\title{
THE EQUALITY OF THE VIRTUAL DELAY AND ATTAINED WAITING TIME DISTRIBUTIONS
}

\author{
HIROTAKA SAKASEGAWA, ${ }^{*}$ University of Tsukuba \\ RONALD W. WOLFF, ${ }^{* *}$ University of California, Berkeley
}

\begin{abstract}
It has recently been shown that for the $G / G / 1$ queue, virtual delay and attained waiting time have the same stationary distribution. We present a sample-path derivation of this result.
\end{abstract}

G/G/1 QUEUE; FIFO; FCFS; WORK IN SYSTEM

Sengupta (1989) has recently shown that for the first-in-first-out (FIFO) $G / G / 1$ queue, virtual delay and attained waiting time (defined below) have the same stationary distribution. His proof is based on relationships between stationary quantities in Miyazawa (1979), (1983).

In this paper, we present a simple and direct sample-path proof of this result. In fact, our result is more general because it depends only on the existence and relative magnitude of two limits. In what follows, we fix a point $\omega$ in the sample space, and treat all quantities as numbers.

Let customer $C_{n}$ arrive at epoch $t_{n}$ and have service time $S_{n}, n \geqq 1$, where $0 \leq t_{1} \leqq t_{2} \cdots$. Customers are served at a single-server FIFO queue. Let virtual delay (work in system) $V(t)$ be the sum of the remaining service times of all customers in system at epoch $t \geqq 0$, and $V_{n}$ be the work found by $C_{n}$, which is the sum of the remaining service times of all customers $C_{j}$, $j<n$, at epoch $t_{n}$. If customers arrive one at a time, $V_{n}=V\left(t_{n}^{-}\right)$. For arbitrary fixed $V(0)$, $V_{1}=\left(V(0)-t_{1}\right)^{+}$.

For every fixed $V(0)$, assume that the system empties infinitely often. A sufficient condition for this is that the following limits exist:

$$
\lim _{n \rightarrow \infty} t_{n} / n=1 / \lambda \text { and } \lim _{n \rightarrow \infty} \sum_{j=1}^{n} S_{j} / n=1 / \mu \text {, where } 0<\lambda<\mu<\infty .
$$

Now $C_{n}$ enters service at epoch $\tau_{n}=t_{n}+V_{n}$, and we define the attained waiting time $W_{a}(t)$ of the customer in service at epoch $t$ as

$$
W_{a}(t)=t-t_{n}=V_{n}+t-\tau_{n} \text { for } t \in\left[\tau_{n}, \tau_{n}+S_{n}\right),
$$

which is the work found by $C_{n}\left(C_{n}\right.$ 's delay in queue $)$ plus $C_{n}$ 's attained service at epoch $t$. Define $W_{a}(t)=0$ if the system is empty epoch $t$.

Notice that the area under $\left\{W_{a}(t)\right\}$ while $C_{n}$ is in service is a trapezoid with base $S_{n}$, left side $V_{n}$, and right side $V_{n}+S_{n}$. We find a corresponding area under $\{V(t)\}$. For this purpose, we pretend that the queue is operated under preemptive LIFO (PL), where the server is always serving the most recent arrival (the customer $C_{n}$ with the largest subscript), among those in system. PL has no effect on the sample paths of $\{V(t)\}$. Under PL, consider the area

Received 10 August 1989.

* Postal address: Institute of Socio-Economic Planning, The University of Tsukuba, Tsukuba, Ibaraki 305, Japan.

${ }^{* *}$ Postal address: Department of Industrial Engineering and Operations Research, University of California, Berkeley, CA 94720, USA.

Part of this research was done while R. W. Wolff visited the Department of Information Sciences, Science University of Tokyo, in 1989. 
under $\{V(t)\}$ while $C_{n}$ is in service. Because service on $C_{n}$ may be interrupted, this area may have disjoint pieces. Put the pieces together! We again have a trapezoid with the same base $S_{n}$, but now the right side is $V_{n}$, and the left side is $V_{n}+S_{n}$. It follows immediately that while $C_{n}$ is in service (under FIFO for $W_{a}$ and PL for $V$ ), the amount of time for which $V(t)>x$ is equal to the amount of time for which $W_{a}(t)>x$. Adding up these amounts, we have the following result.

Theorem 1. For every busy period and every $x \geqq 0$, the amount of time for which $V(t)>x$ is equal to the amount of time for which $W_{a}(t)>x$.

To convert Theorem 1 into a statement about time averages, let $\left\{W_{a}^{\prime}(t)\right\}$ be the process we get when the 'high' and 'low' sides of the trapezoids under $\left\{W_{a}(t)\right\}$ are reversed, and let $I_{v x}(t)$, $I_{w x}(t)$, and $I_{w x}^{\prime}(t)$ be the indicators of the events $V(t)>x, W_{a}(t)>x$, and $W_{a}^{\prime}(t)>x$, respectively; $t, x \geqq 0$.

We now compare the integrals $\int_{0}^{t} I . .(u) d u$. The processes $V$ and $W_{a}^{\prime}$ have the same trapezoids, where the trapezoid of $C_{n}$ begins earlier for $V$, and may end later, but only if work exceeds $V_{n}$ in between. Thus the contribution of $C_{n}$ to $I_{v x}$ on $[0, t]$ either occurs earlier, or the work exceeds $x$ throughout $\left[t_{n}, t\right]$. The trapezoids under $W$ and $W_{a}^{\prime}$ are in the same locations, with the high sides under $W_{a}^{\prime}$ on the left. It follows immediately that

$$
\int_{0}^{t} I_{v x}(u) d u \geqq \int_{0}^{t} I_{w x}^{\prime}(u) d u \geqq \int_{0}^{t} I_{w x}(u) d u .
$$

Because the completed portions of the trapezoids under $V$ in the interval $\left[0, t-W_{a}(t)\right]$ correspond to customers who arrived earlier than the (FIFO) customer in service at epoch $t$, we have the left-hand inequality in (4), which is combined with (3), for $t>0$ and $x \geqq 0$,

$$
\int_{0}^{t-W_{a}(t)} I_{v x}(u) d u / t \leqq \int_{0}^{t} i_{w x}(u) d u / t \leqq \int_{0}^{t} I_{v x}(u) d u / t .
$$

Now let $t \rightarrow \infty$ in (4). Both processes have the same time average, when the limits exist, provided that

$$
W_{a}(t) / t \rightarrow 0 \text { as } t \rightarrow \infty .
$$

We show that (1) implies (5) by first obtaining a result for the unstable system obtained by adding constant $c=1 / \lambda-1 / \mu+\varepsilon \geqq 0$ to all of the service times. Let $V_{n c}$ be the work found by $C_{n}$ with these altered service times. Let $X_{n}=S_{n}+c-\left(t_{n+1}-t_{n}\right)$, where $\lim _{n \rightarrow \infty} \sum_{j=1}^{n} X_{j} / n=\varepsilon$.

Now $\left\{V_{n c}\right\}$ is simply the standard delay-in-queue sequence for a single-server queue, where

$$
V_{n+1, c}=\left(V_{n c}+X_{n}\right)^{+}, \text {and } Y_{n}=\left(V_{n c}+X_{n}\right)^{-}, \quad n \geqq 1,
$$

where $Y_{n}$ is the idle time of the server between the completion of service on $C_{n}$ and the commencement of service on $C_{n+1}$. From (6), we can write

$$
V_{n+1, c}=V_{1}+\sum_{j=1}^{n} x_{j}+\sum_{j=1}^{n} Y_{j}, \quad n \geqq 1 .
$$

If $c$ is large enough to make $\varepsilon>0$, there is an $m$ such that $\sum_{j=1}^{n} X_{j}>0$ for all $n \geqq m$, which means that the queue remains busy from that point on, $Y_{n}=0$ for all $n>m$, and we can write

$$
V_{n+1, c}=V_{1}+\sum_{j=1}^{n} X_{j}+\sum_{j=1}^{m} Y_{j}, \quad n \geqq m .
$$

Now divide (7) by $n$, and let $n \rightarrow \infty$. We have

$$
\lim _{n \rightarrow \infty} V_{n c} / n=\varepsilon>0 \text {, }
$$

where $\varepsilon$ may be arbitrarily small. 
Now the $V_{n c}$ are monotone non-decreasing in $c$ for every $n$, and it follows immediately from (8) that when the limits in (1) hold under the slightly weaker conditions $0<\lambda \leqq \mu<\infty$,

$$
\lim _{n \rightarrow \infty} V_{n} / n=0 .
$$

Remark. Our argument for (8) and (9) is similar to that in Loynes (1962). His results are in a stochastic setting, where $\left\{X_{n}\right\}$ is stationary and ergodic, but his approach is really sample-path in nature.

For $t>0$, let $C_{n(t)}$ be the customer in service at epoch $t$ under FIFO, and write

$$
W_{a}(t) / t \leqq\left(V_{n(t)}+S_{n(t)}\right) / t_{n(t)},
$$

where if the system is empty at epoch $t$, we set $t_{n(t)}=t$, and the other quantities in (10) equal to 0 . Now $n(t) \rightarrow \infty$ as $t \rightarrow \infty$, and from (1), $t_{n(t)} / n(t) \rightarrow 1 / \lambda$ as $t \rightarrow \infty$, and $S_{n} / n \rightarrow 0$ as $n \rightarrow \infty$. Thus, from (9) and (10), it is easy to see that (1) implies (5).

From (4) and that (1) implies (5), we have the following result.

Theorem 2. When (1) holds, the fraction of time that $\{V(t)\}$ exceeds $x$ is equal to the fraction of time that $\left\{W_{a}(t)\right\}$ exceeds $x$, for every $x \geqq 0$, whenever these time averages exist as limits. If one time average exists, so does the other.

In a stochastic setting, where these time averages exist as constants w.p.1, and each process has a stationary distribution, each distribution must equal the correponding time average. Hence, in the stochastic setting in Sengupta (1989), Theorem 2 is equivalent to his result. Also note that in our analysis, we do not require either arrivals or departures to be one at a time.

Not only is our analysis more general, we also have learned a great deal about the close connection of these processes on finite intervals.

\section{References}

LOYNEs, R. M. (1962) The stability of a queue with non-independent inter-arrival and service times. Proc. Camb. Phil. Soc. 58, 497-520.

MiYazAWA, M. (1979) A formal approach to queueing processes in the steady state and their applications. J. Appl. Prob. 16, 332-346.

MiYAZAWA, M. (1983) The derivation of invariance relations in complex queueing systems with stationary inputs. Adv. Appl. Prob. 15, 874-885.

SEnguPtA, B. (1989) An invariance relationship for the $G / G / 1$ queue. Adv. Appl. Prob. 21, 956-957. 